\title{
The primary energy spectrum with the Linsley method correcting heavy primary effects in the LAAS experiments
}

\author{
Hiroki Matsumoto ${ }^{1, \mathrm{a}}$, Atsushi Iyono ${ }^{2, \mathrm{~b}}$, Kazuhide Okei ${ }^{1}$, Shuhei Tsuji ${ }^{1}$, Nobuaki Ochi ${ }^{3}$, Nobusuke Takahashi ${ }^{4}$, \\ and Saya Yamamoto ${ }^{2}$ \\ 1 Kawasaki Medical School, Kurashiki 701-0192, Japan \\ 2 Dept. of Fundamental Science, Faculty of Science, Okayama University of Science, Okayama 700-0005, Japan \\ 3 Toyo University, Tokyo 112-8606, Japan \\ ${ }^{4}$ Dept. of Physics, Hirosaki University, Hirosaki 036-8560, Japan
}

\begin{abstract}
The compact EAS Extensive Air Shower (EAS) array $\left(200 \mathrm{~m}^{2}\right)$ and the Linsley method have been used with the aim to observe the primary energy spectrum. The spectral indexes $\alpha_{\text {proton }}$ and $\alpha_{\text {iron }}$ are obtained as $-2.46 \pm 0.17$ and $-3.08 \pm 0.24$, respectively, in the primary energy region of $10^{16} \mathrm{eV}-10^{18.5} \mathrm{eV}$ in the single observation of the compact EAS array. Additionally, $\alpha_{\text {proton }}$ and $\alpha_{\text {iron }}$ are obtained as $-3.12 \pm 0.13$ and $-3.52 \pm 0.17$, respectively, in the same primary energy region in the observation by restricting the zenith angle of EAS events.
\end{abstract}

\section{Introduction}

The Large Area Air Shower (LAAS) experiments [1,2] have been observing cosmic rays with compact Extensive Air Shower (EAS) arrays, and have maintained EAS arrays at several institutes since 1996. The purposes of LAAS experiments are to study the primary energy spectrum, anisotropy of high energy cosmic rays, and simultaneous and parallel EAS events [3-7]. The group of Okayama University of Science has mainly observed cosmic rays in the primary energy range above $10^{15} \mathrm{eV}$ and studied its primary energy spectrum.

In order to observe EASs caused by high energy cosmic rays on the ground, many detectors scattered over a large area are required such as the Pierre Auger Observatory [8] and the Telescope Array Observatory [9] due to the feature of primary energy spectrum. However, these methods have a high cost in installing and maintaining detectors.

To solve the problem and carry out low-cost observation, we adapted the Linsley method [10], which is the result of the EAS core distance, $r$, dependent on the dispersion, $\sigma_{t}$, of the arrival time of EAS particles to the compact EAS array in LAAS experiments. Bezboruah [11] has also reported on the results of the primary energy spectrum obtained by adapting the Linsley method to their mini-array.

In the LAAS experiments, the observation of the primary energy spectrum has been carried out by combining Linsley's method with the compact EAS array since 2006, and an apparatus for improving the determination accuracy of the primary energy was installed in 2008 .

\footnotetext{
a e-mail: h.matsumoto@med.kawasaki-m.ac.jp

b e-mail: iyono@das.ous.ac.jp
}

\section{Apparatus}

Okayama University of Science (OUS) is in Okayama, Japan and located at $34^{\circ} 42^{\prime}(\mathrm{N})$ and $133^{\circ} 56^{\prime}(\mathrm{E})$, at sea level. There are five EAS arrays (OUS1 OUS5) and these arrays, except the OUS4 which is the apparatus for obtaining information of the EAS zenith angle, each consist of eight scintillation counters.

The arrangement of the OUS1 array is shown in Fig. 1. In this work, the obtained data of OUS1 and OUS4 are used. Each scintillation counter equips a plastic scintillator of size $50 \mathrm{~cm} \times 50 \mathrm{~cm} \times 5 \mathrm{~cm}$, a PMT (HAMAMATSU H7195) and a stainless steel case of 5 $\mathrm{mm}$ thickness. The OUS1 array is installed on the rooftop of a building in Okayama University of Science, covers an area of about $200 \mathrm{~m}^{2}$. The data acquisition system (DAQ) consists of a CAMAC TDC (Kaizuworks Model 3781), a CAMAC ADC (Lecroy Model 2249W), and a shift register (Mbeware Model PL-320E). In this work, we use EAS event data obtained by the shift register because the shift register allows storing more information of time and the number of EAS singles from detectors than the TDC system. The event trigger time and clock synchronization are maintained by a GPS module (Kaizuworks Model 3051A) of time accuracy $1 \mu \mathrm{s}$. An EAS event is triggered by a coincidence detection of more than three detectors within a $2.5 \mu$ s time window.

The shift register module is able to store the time series signal from each detector as digital data with $\pm 2.5 \mu$ s time window from an EAS trigger time. The time resolution of the shift register is $5 \mathrm{~ns}$.

OUS4 is the apparatus for obtaining the zenith angle of EAS events and is shown in Fig. 1. This apparatus equips scintillation counters for the EAS event trigger on the top and bottom layer, respectively, and for anti-counting at the side panels. The scintillation counter, the size of which is $20 \mathrm{~cm} \times 50 \mathrm{~cm} \times 1 \mathrm{~cm}$, for the event trigger, is equiped 

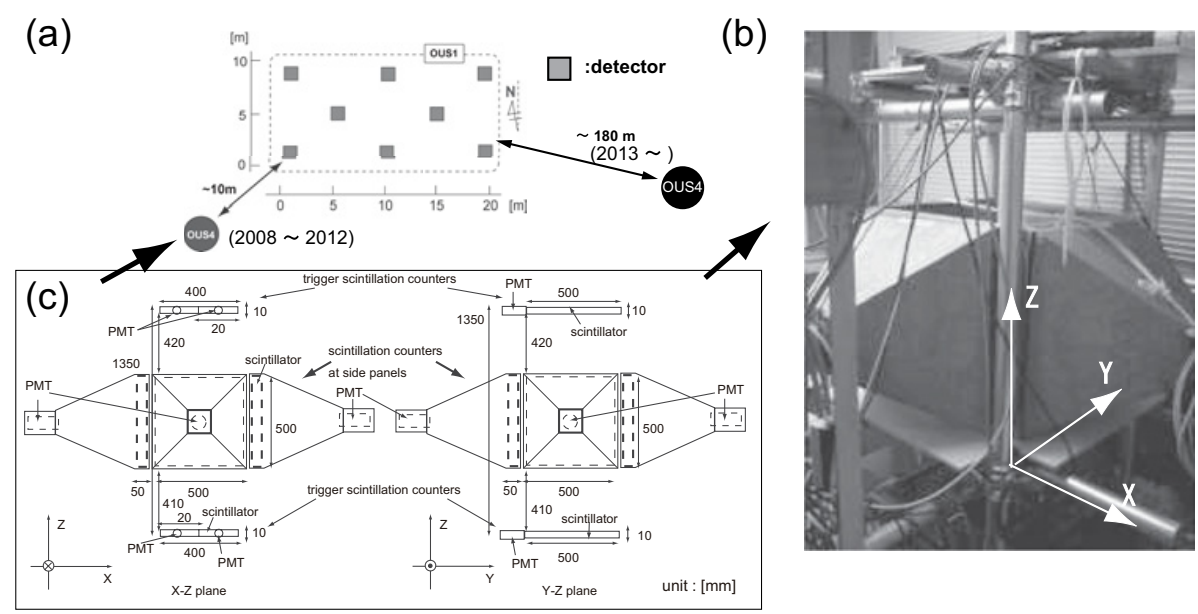

Figure 1. (a) The arrangement of OUS1 and OUS4. (b) Photograph of OUS4. (c) The schematic drawing of OUS4.

with a PMT and a black plastic cover. The total area of each of the bottom and top counters is $0.2 \mathrm{~m}^{2}$ respectively. Anti-counters at the side panels are the same as those of OUS1. DAQ is also the same as the OUS1 and the GPS module is equipped for synchronizing the observation clock. The zenith angle, $\theta$, of EAS events is distinguished by using anti-counters events of $\theta<25.6^{\circ}$ from all events. The OUS4 was located at about a distance of $10 \mathrm{~m}$ from the OUS1 in $2008 \sim 2012$. In 2013, we relocated OUS4 to a distance of $180 \mathrm{~m}$ from OUS1.

\section{Method}

\subsection{EAS Core distance}

The estimation of the core distance, $r$, of EAS is performed by using Linsley's method and the result of Okita [6]. Linsley showed that the relation between the average dispersion of arrival time distribution of EAS particles, $\left\langle\sigma_{\mathrm{t}}\right\rangle$, and $r$ follows the empirical formula

$$
\left\langle\sigma_{t}\right\rangle=\sigma_{t 0}\left(1+\frac{r}{r_{t}}\right)^{b},
$$

where

$$
\begin{gathered}
b=(2.08 \pm 0.08)-(0.4 \pm 0.06) \sec \theta \\
+(0 \pm 0.06) \log \left(E / 10^{17} \mathrm{eV}\right)
\end{gathered}
$$

$\sigma_{t 0}=1.6 \mathrm{~ns}, r_{t}=30 \mathrm{~m}, \theta$ is the zenith angle and $E$ is the primary cosmic ray energy. We adapt $b$ to the averaged value $\bar{b}=1.65$ by the EAS zenith angle distribution expected by the observation instead of the original $b$, and ignore the contribution of the primary energy. In Fig. 2, $\left\langle\sigma_{t}\right\rangle$ is plotted as a function of $r$.

The EAS arrival distribution is assumed to be the gamma distribution by Linsley, and the best estimator, discussed in reference [6], of the dispersion of arrival time distribution of EAS particles, $\sigma_{t}$, is

$$
\sigma_{\mathrm{t}}=\frac{\sqrt{2}}{1.67} t_{\mathrm{m}},
$$

where $t_{\mathrm{m}}$ is the median of the time series data of the observed EAS events. The core distance, $r$, is obtained by

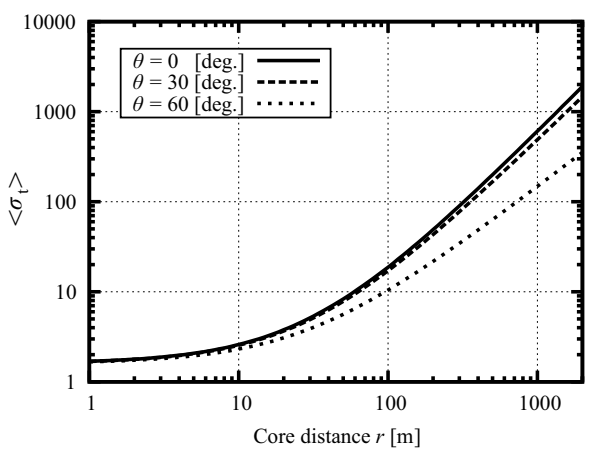

Figure 2. The relation between $r$ and $\left\langle\sigma_{t}\right\rangle$. Each curve is Eq. (1) for $\theta=0^{\circ}, 30^{\circ}, 60^{\circ}$, respectively. The averaged $\theta$ is $\sim 23^{\circ}$ in OUS1.

substitution of Eq. (1) into Eq. (3), and rearrangement of the terms

$$
r=30\left(\left(1.35 t_{\mathrm{m}}\right)^{\frac{1}{1.65}}\right)
$$

Note that the systematic error of $r$ increases with increasing EAS zenith angle $\theta$, because we use the averaged $\bar{b}$ instead of the original $b$.

\subsection{Primary energy}

In order to determine the primary energy of EAS events, we combine the database of lateral distributions of muons and electrons obtained by carrying out the EAS simulation program AIRES, and the observed data of the core distance and the particle density. In the EAS simulation, hadronic interaction models are assumed to be QGSJET II-3 and the Hillas Splitting model. The primary nuclei are assumed to be protons and irons, respectively. This simulation procedure is described in Ref. [7] in detail.

Figure 3 shows the calculated energy distribution assumed to be that the input primary energy $E_{0}$ is at $10^{17} \mathrm{eV}$ in the primary nuclei of protons and irons in the simulation. The OUS1+4 in Fig. 3-(B) indicates the synchronized observation between OUS1 and OUS4. By restricting the zenith angle of EAS events by OUS4, the accuracy of the determination of the primary energy of OUS1+4 is able to improve more than that of the OUS1. 

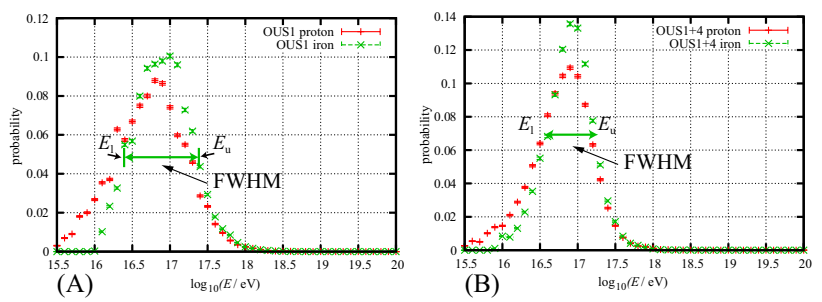

Figure 3. The simulated energy distributions in OUS1 (A) and OUS1+4 (B). Symbols of $(+)$ and $(*)$ indicate proton primaries and iron ones, respectively.

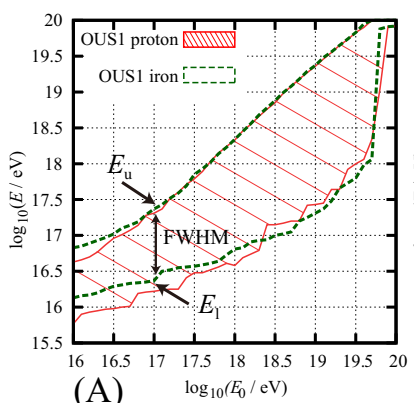

(A)

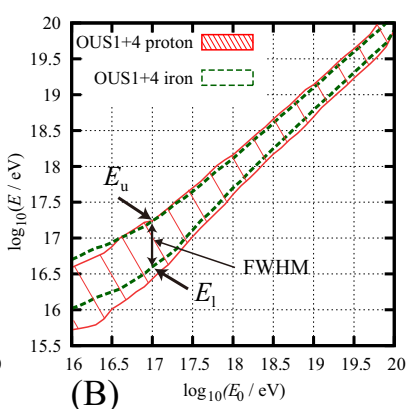

(B)
Figure 4. The energy resolution bands in the OUS1 (A) and the OUS1+4 (B). The shaded bands indicate the result of assumption of proton primaries. The dashed line bands indicate the result of assumption of iron primaries.

We define the energy resolution of detectors as the FWHM value of the calculated energy distribution. Figure 4 shows that the relation between $E_{0}$ and the FWHM. $E_{1}$ and $E_{\mathrm{u}}$ in Fig. 4 correspond to the same letters in Fig. 3, respectively.

In order to determine the primary energy spectrum, the observed data spectrum corresponds to the spectrum calculated by the detector simulation. The true primary spectrum is assumed to be a single power-law $E^{\alpha}$, where $\alpha$ is the spectral index value in the simulation. The $\alpha$ value is obtained as $\alpha^{\prime}$ by being affected by the detector sensitivity and the fluctuation of the shower size. The calculation method of $\alpha$ from $\alpha^{\prime}$ is described in Refs. [7] and [12].

\section{Result}

\subsection{Data analysis}

In this analysis, the data period is from 4/2006 to $3 / 2016$ for OUS1, and from 8/2008 to 12/2012 in the synchronized observation between OUS1 and OUS4 (OUS1+4). The observation time, the total number of events and the number of events enabled to determine the primary energy are summarized in Table 1 . The assumption of the primary nuclei are protons and irons, and $N_{\text {proton }}$ and $N_{\text {iron }}$ indicate the number of events enabled to determine the primary energy in each assumption, respectively.

The selection criteria for available events are the range of the observed core distance $r_{\text {obs }}, 100 \mathrm{~m}<r_{\text {obs }}<2000 \mathrm{~m}$. Additionally, in OUS1+4, we used the coincidence events of the time difference between OUS1 and OUS4 within $10 \mu \mathrm{s}$, and the events of the EAS zenith angle $\theta$ within $\theta<25.6^{\circ}$.
Table 1. The data summary of the OUS1 and OUS4 observations.

\begin{tabular}{|l|c|c|c|c|}
\hline & $T_{\text {obs }}[$ day] & $N_{\text {obs }}$ & $N_{\text {proton }}$ & $N_{\text {iron }}$ \\
\hline \hline OUS1 & 2171 & $1.5 \times 10^{7}$ & $8.6 \times 10^{4}$ & $1.8 \times 10^{5}$ \\
\hline OUS1+4 & 1336 & $2.0 \times 10^{5}$ & $5.5 \times 10^{2}$ & $1.9 \times 10^{3}$ \\
\hline
\end{tabular}

$T_{\text {obs }}$ : Total observation time

$N_{\text {obs }}$ : Total number of triggered events

$N_{\text {proton }}$ : Number of events enabled to determine primary energy $\left(\geq 10^{16} \mathrm{eV}\right)$ assumed nuclei to be protons

$N_{\text {iron }}$ : Number of events enabled to determine primary energy $\left(\geq 10^{16} \mathrm{eV}\right)$ assumed nuclei to be iron.

Table 2. The spectral index $\alpha^{\prime}$ and $\alpha$.

\begin{tabular}{|l|c|c|c|c|}
\hline \multirow{2}{*}{\multicolumn{2}{|c|}{}} & \multicolumn{3}{|c|}{ Primary energy region [eV] } \\
\cline { 2 - 5 } \multicolumn{2}{l|}{} & (i) $10^{16}-10^{19.5}$ & (ii) $10^{16}-10^{18.5}$ & (iii) $10^{18}-10^{19.5}$ \\
\hline \hline \multirow{2}{*}{$\begin{array}{l}\text { OUS1 } \\
\text { Proton }\end{array}$} & $\alpha^{\prime}$ & $-2.37 \pm 0.11$ & $-2.62 \pm 0.15$ & $-2.09 \pm 0.18$ \\
\cline { 2 - 5 } $\begin{array}{l}\text { OUS1 } \\
\text { Iron }\end{array}$ & $-2.19 \pm 0.11$ & $-2.46 \pm 0.17$ & $-1.93 \pm 0.17$ \\
\cline { 2 - 5 } & $\alpha$ & $-2.61 \pm 0.13$ & $-2.96 \pm 0.15$ & $-2.07 \pm 0.07$ \\
\hline \hline \multirow{2}{*}{$\begin{array}{l}\text { OUS1+4 } \\
\text { Proton }\end{array}$} & $\alpha^{\prime}$ & $-2.92 \pm 0.18$ & $-3.08 \pm 0.24$ & $-2.00 \pm 0.07$ \\
\cline { 2 - 5 } $\begin{array}{l}\text { OUS1+4 } \\
\text { Iron }\end{array}$ & $\alpha^{\prime}$ & $-3.87 \pm 0.16$ & $-3.14 \pm 0.25$ & - \\
\cline { 2 - 5 } & $\alpha$ & $-3.23 \pm 0.12 \pm 0.13$ & $-3.47 \pm 0.15$ & - \\
\hline
\end{tabular}

\subsection{Primary energy spectrum}

The primary energy spectra obtained by this analysis are shown in Fig. 5. In order to calculate the spectral index value of the data spectrum, each spectrum is fitted by the least squares method to a single power-law spectrum, $E^{\alpha^{\prime}}$. Because the spectral index seems to manifest a flattening around $10^{18.5} \mathrm{eV}$, the primary energy regions of the fitting are

$$
\begin{aligned}
& \text { (i) } 10^{16} \mathrm{eV} \sim 10^{19.5} \mathrm{eV} \text {, } \\
& \text { (ii) } 10^{16} \mathrm{eV} \sim 10^{18.5} \mathrm{eV} \text { and } \\
& \text { (iii) } 10^{18} \mathrm{eV} \sim 10^{19.5} \mathrm{eV} .
\end{aligned}
$$

The OUS1 obtained spectral indexes $\alpha_{\text {proton }}^{\prime}$ and $\alpha^{\prime}$ iron $-2.62 \pm 0.15$ and $-2.96 \pm 0.15$, respectively, in the primary energy region (ii). Thus, $\alpha_{\text {proton }}$ and $\alpha_{\text {iron }}$ are equal to $-2.46 \pm 0.17$ and $-3.08 \pm 0.24$, respectively, by calculating from the relation between $\alpha$ and $\alpha^{\prime}$.

In OUS $1+4, \alpha$ is also calculated by using the same procedure. $\alpha_{\text {proton }}^{\prime}$ and $\alpha_{\text {iron }}^{\prime}$ are $-3.14 \pm 0.25$ and $-3.47 \pm 0.15$, respectively, in the primary energy region (ii), and $\alpha_{\text {proton }}$ and $\alpha_{\text {iron }}$ are equal to $-3.12 \pm 0.13$ and $-3.52 \pm 0.17$, respectively. The obtained spectral indexes are summarized in Table 2.

\section{Conclusion}

In the LAAS experiments, we have observed cosmic rays and studied various phenomena attributed by those with compact EAS arrays since 1996. Especially, the primary energy spectrum has been observed by adapting Linsley's method to our compact EAS array in Okayama University of Science since 2008. The primary energy spectrum is updated in this work, $\alpha_{\text {proton }}$ and $\alpha_{\text {iron }}$ are equal to $-2.46 \pm 0.17$ and $-3.08 \pm 0.24$, respectively, in the primary energy region of $10^{16} \mathrm{eV}-10^{18.5} \mathrm{eV}$ in the 

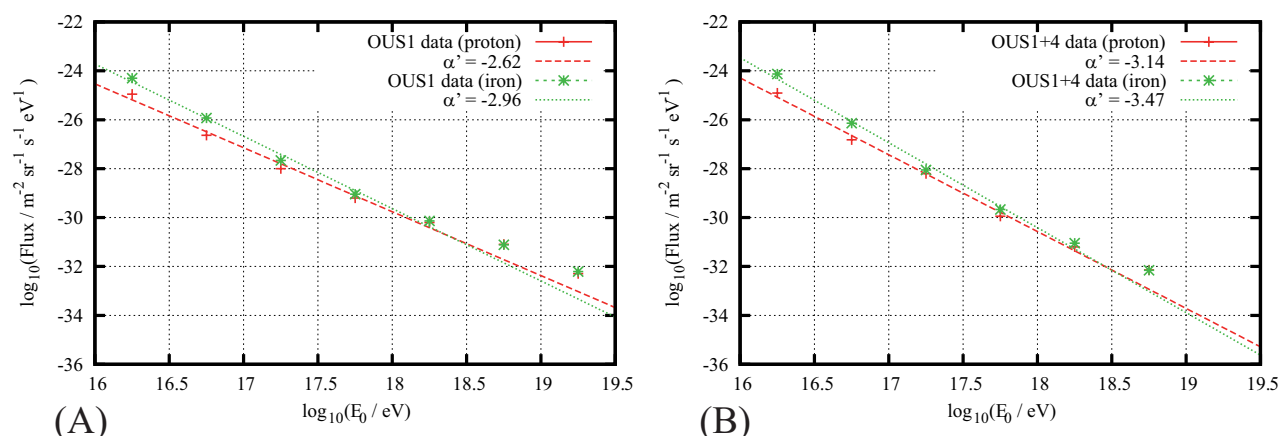

Figure 5. The data spectrum assumed to be proton and iron primaries, respectively, in the OUS1 (A), and in the OUS1+4 (B). Symbols of $(+)$ and $(*)$ indicate observed data. Lines show the result of the least square fittings.

OUS1. also, $\alpha_{\text {proton }}$ and $\alpha_{\text {iron }}$ are equal to $-3.12 \pm 0.13$ and $-3.52 \pm 0.17$, respectively, in the same primary energy region in OUS1+4.

\section{References}

[1] T. Wada et al., Nucl. Phys. B (Proc. Suppl.) 75, issue 1-2, 330-332 (1999)

[2] N. Ochi et al., J. Phys. G: Nucl. Part. Phys. 29, 1169-1180 (2003)

[3] A. Iyono et al., Proceedings of the 30th ICRC Merida, 114 (2007)

[4] S. Ohara et al., Proceedings of the 32nd ICRC Beijing, 406 (2011)

[5] A. Iyono et al., Astrophysics and Space Sciences Transactions 7, 327-333 (2011)
[6] M. Okita et al., Nucl. Phys. B (Proc. Suppl.) 175-176, 322-325 (2008)

[7] H. Matsumoto et. al., Nucl. Instr. \& Meth. A 614, 475-482 (2010)

[8] I. Valino, Proceedings of the 34th ICRC Hague, 271 (2015)

[9] C. Jui, Proceedings of the 34th ICRC Hague, 35 (2015)

[10] J. Linsley, J. Phys. G: Nucl. Phys. 12, 51-57 (1986)

[11] Bezboruah et al., Astropart. Phys. 11, 395 (1996)

[12] H. Matsumoto et. al., Proceedings of the 34th ICRC Hague, 257 (2015)

[13] S. J. Sciutto, AIRES (1999) [astro-ph/9911331]

[14] S. Ostapchenko, Phys. Rev. D 74, 014026 (2006)

[15] A. M. Hillas, Nucl. Phys. B (Proc. Suppl.) 52, issue 3, 29-42 (1997) 